\title{
Inertial particles driven by a telegraph noise
}

\author{
G. Falkovich, ${ }^{1}$ S. Musacchio, ${ }^{1}$ L. Piterbarg, ${ }^{2}$ and M. Vucelja ${ }^{1}$ \\ ${ }^{1}$ Weizmann Institute of Science, Rehovot, 76100 Israel \\ ${ }^{2}$ University of Southern California, Los Angeles, California 90089, USA
}

(Received 29 March 2007; published 22 August 2007)

\begin{abstract}
We present a model for the Lagrangian dynamics of inertial particles in a compressible flow, where fluid velocity gradients are modeled by a telegraph noise. The model allows for an analytic investigation of the role of time correlation of the flow in the aggregation-disorder transition of inertial particles. The dependence on the Stokes number St and the Kubo number $\mathrm{Ku}$ of the Lyapunov exponent of particle trajectories reveals the presence of a region in parameter space $(\mathrm{St}, \mathrm{Ku})$, where the leading Lyapunov exponent changes sign, thus signaling the transition. The asymptotics of short- and long-correlated flows are discussed, as well as the fluid-tracer limit.
\end{abstract}

DOI: 10.1103/PhysRevE.76.026313

PACS number(s): 47.52. $+\mathrm{j}, 47.55 . \mathrm{Kf}, 47.27 . \mathrm{eb}$

\section{INTRODUCTION}

The spontaneous formation of clusters of particles suspended in chaotic flows may originate from two different physical processes: compressibility of the fluid flow and particle inertia. In the first case particles are trapped in regions of ongoing compression, while in the second case inertia causes their ejection from vortical regions. The underlying link between these two phenomena is manifested in the limit of weak inertia, in which particle dynamics in incompressible flows can be approximated by that of tracers in a weakly compressible velocity field $[1,2]$.

Clustering processes have been extensively studied, both in the case of compressible flows [3-6] and inertial particles [2,7-9]. Fractal dimension of the clusters, obtained from the ratios of Lyapunov exponents of particle trajectories, has revealed a powerful tool to quantify the intensity of the clustering [3,7], and has motivated further studies of Lyapunov exponents of inertial particles $[9,10]$.

Aggregation is an extreme form of clustering, which occurs when the trajectories of different particles tend to pointlike clusters. In this situation the senior Lyapunov exponent of particle trajectories become negative, signaling that nearby particles do not separate exponentially as expected in chaotic flows, but their paths eventually coalesce. This phenomenon has been described for fluid tracers in compressible flows $[11,12]$, as well as for light particles in incompressible flows [7].

Surprisingly, it has been shown that in compressible flows, where fluid trajectories coalesce, large enough inertia can induce a transition from the strong clustering regime into a weak clustering one, where particle trajectories remain chaotic and the senior Lyapunov exponent is positive [13-16]. Analytic results on this aggregation-disorder transition have been obtained under the assumption that the carrier flow is short correlated in time.

It is therefore natural to ask how time correlations of real turbulent flows can influence this phenomena. As pointed out by recent numerical studies of fluid tracers in compressible flows [6], time correlations are responsible for an increase in the level of compressibility required for the transition to the strong-clustering regime. In the case of inertial particles it has been found [10] that time correlations cause an increase of the (positive) Lyapunov exponent (i.e., make particle flow more chaotic) already at weak inertia, which cannot be predicted in the framework of short-correlated flows $[9,17-19]$.

Here we discuss a simple one-dimensional, timecorrelated model flow, recently introduced in [20], which allows one to obtain a deeper insight on the interplay between inertia and compressibility, and to investigate analytically the dependence on Stokes and Kubo numbers of the Lyapunov exponent of particle trajectories.

\section{MODEL}

The dynamics of two small inertial particles, whose density is much larger than the fluid density, is dominated by the viscous drag. Hence, the equation for their separation $\mathbf{R}(t)$ $=\mathbf{X}_{1}(t)-\mathbf{X}_{2}(t)$ and relative velocity $\mathbf{V}$ reads [21]

$$
\dot{\mathbf{R}}=\mathbf{V}, \quad \dot{\mathbf{V}}=-\frac{1}{\tau}\left[\mathbf{V}-\Delta \mathbf{U}\left(\mathbf{X}_{1}, \mathbf{X}_{2}, t\right)\right],
$$

where $\tau$ is the Stokes time of the particles, and $\Delta \mathbf{U}$ is the difference of fluid velocities at particle positions.

To investigate the behavior of the Lyapunov exponent one has to consider separations smaller than the viscous length scale of the flow. At these scales the fluid velocity difference can be written in terms of the velocity gradients $S_{i j}=\partial_{j} U_{i}$ as $\Delta U_{i}=S_{i j} R_{j}$. The Lyapunov exponent $\lambda$ is determined by the contributions of the local gradients $S_{i j}(t)$ experienced by the two nearby trajectories. Note that the statistics of velocity gradients in the reference frame of an inertial particle differs from the Eulerian statistics due to preferential concentration.

Let us now consider an idealized one-dimensional flow, in which the fluid velocity gradient in the reference frame of a particle is modeled by a telegraph noise $s(t)$, i.e., a noise that switches randomly between two fixed values $\pm s$. Stokes number can be defined in term of the fluid gradient intensity as $\mathrm{St}=s \tau$. Equations (1) reduce to

$$
\dot{R}=V, \quad \dot{V}=-\frac{V}{\tau}+\frac{s(t)}{\tau} R .
$$

When $s(t)=+s$ the system is expanding, and evolves towards the asymptote $V=\alpha R$, where 


$$
\alpha=\frac{1}{2 \tau}(-1+\sqrt{1+4 s \tau}) .
$$

The particles separate exponentially with smaller rate than fluid trajectories $\alpha<s$. When $s(t)=-s$ the system is contracting, and two different scenarios are observed, according to the relative intensity of fluid gradients and inertial drag. For small Stokes numbers $\mathrm{St}<1 / 4$ the system evolves toward the asymptote $V=\beta R$, where

$$
\beta=\frac{1}{2 \tau}(-1+\sqrt{1-4 s \tau}) \quad \text { for } s \tau<1 / 4
$$

Particles slow down less efficiently than the fluid, and hence their separation goes to zero with a faster exponential rate $|\beta|>s$. For large Stokes number $\mathrm{St}>1 / 4$ the system has two complex conjugate eigenvalues

$$
\beta=\frac{1}{2 \tau}(-1 \pm i \sqrt{4 s \tau-1}) \quad \text { for } s \tau>1 / 4,
$$

and the solution decays exponentially with a clockwise spiral motion in phase space $(R, V)$. This means that particles can cross the $R=0$ axis, i.e., collide, with nonzero relative velocity, giving origin to a shock [22]. Notice that the solution is properly defined also for $R<0$. The change of the sign of $R$ can be interpreted as the fast particle overcoming the slow one.

Shocks start to appear for the critical value $\mathrm{St}^{*}=1 / 4$. The presence of such critical Stokes number is characteristic of flows where the fluid velocity gradients are bounded, and its value is determined by the intensity of the strongest negative gradient (here $-s$ ). Conversely, if the statistics of the fluid velocity gradient is unbounded, shocks can appear for arbitrarily small Stokes number, but they are exponentially suppressed in the limit $\mathrm{St} \rightarrow 0[18,22,23]$.

The transition rates $\nu_{1}$ from $-s$ to $s$ and $\nu_{2}$ from $s$ to $-s$ determine the fraction of time spent by the particle pair in regions of ongoing compression and expansion (respectively, $\nu_{2} /\left(\nu_{1}+\nu_{2}\right)$ and $\left.\nu_{1} /\left(\nu_{1}+\nu_{2}\right)\right)$. The mean value of the noise $s(t)$ is

$$
s_{0} \equiv\langle s(t)\rangle=-s \Delta \nu / \nu,
$$

where $\nu=\nu_{1}+\nu_{2}$ and $\Delta \nu=\nu_{2}-\nu_{1}$. Noise fluctuations $\tilde{s}(t)$ $\equiv s(t)-s_{0}$ are exponentially correlated:

$$
\left\langle\widetilde{s}(t) \widetilde{s}\left(t^{\prime}\right)\right\rangle=\frac{4 s^{2} \nu_{1} \nu_{2}}{\nu^{2}} \exp \left(-\nu\left|t-t^{\prime}\right|\right) .
$$

The ratio between the correlation time of the flow $1 / \nu$ and the Lagrangian separation time $1 / s$ defines the Kubo number $\mathrm{Ku}=s \nu^{-1}$.

Notice that in potential flows both fluid tracers and inertial particles spend more time in regions of a local compression than in expanding regions, and hence $\Delta \nu>0$. The actual value of $\Delta \nu$ can be determined by assuming statistical homogeneity and isotropy of the flow. Thanks to these symmetries, the mean position of a particle is solely determined by its initial position $\langle X(t)\rangle=X_{0}$ and hence the distance $R$ is statistically conserved.
The peculiar nature of the telegraph noise, namely the fact that its square is deterministic, allows one to obtain closed equations for $\langle R(t)\rangle$. Averaging over different realizations of the noise, and using the derivative formula [24]

$$
\frac{d}{d t}\left\langle\xi_{t} F_{t}(\xi)\right\rangle=\left\langle\xi_{t} \frac{d}{d t} F_{t}(\xi)\right\rangle-\nu\left\langle\xi_{t} F_{t}(\xi)\right\rangle,
$$

which hold for a generic noise with zero mean and correlation $\left\langle\xi_{t} \xi_{t^{\prime}}\right\rangle \sim \exp \left(-\nu\left|t-t^{\prime}\right|\right)$, one obtains the linear system

$$
\frac{d}{d t} \mathbf{y}=\frac{1}{\tau} M \mathbf{y},
$$

where

$$
\begin{gathered}
\mathbf{y}=\left[\begin{array}{c}
\langle R\rangle \\
\tau\langle V\rangle \\
\tau\langle\tilde{s} R\rangle \\
\tau^{2}\langle\tilde{s} V\rangle
\end{array}\right], \\
M=\left[\begin{array}{cccc}
0 & 1 & 0 & 0 \\
s_{0} \tau & -1 & 1 & 0 \\
0 & 0 & -\nu \tau & 1 \\
\left(s^{2}-s_{0}^{2}\right) \tau^{2} & 0 & -s_{0} \tau & -(1+\nu \tau)
\end{array}\right] .
\end{gathered}
$$

This linear system allows for a constant solution $\langle R\rangle=R_{0}$ when $s_{0} \nu(1+\nu \tau)+s^{2}=0$, which together with Eq. (6) gives

$$
\Delta \nu=\frac{s}{1+\nu \tau} .
$$

Notice that inertia reduces the trapping of the particle in compressing regions. This effect is encoded in the behavior of the parameter $\Delta \nu$, which is a decreasing function of Stokes time $\tau$, and vanishes in the limit $\tau \rightarrow \infty$.

The positivity of $\nu_{1}$ requires $\Delta \nu<\nu$, and gives an upper bound for the Kubo number achievable in the system:

$$
\mathrm{Ku} \leqslant \mathrm{Ku}^{*}=\frac{2 \mathrm{St}}{\sqrt{1+4 \mathrm{St}}-1} .
$$

For longer correlation time, particles are irreversibly captured by contracting regions. In the limit of fluid tracers $\tau$ $\rightarrow 0$ the constraint becomes $\mathrm{Ku}<1$. The accessible region in $(\mathrm{St}, \mathrm{Ku})$ parameter space is shown in Fig. 1.

\section{STATISTICS OF PARTICLE-VELOCITY GRADIENTS}

From the system (2) one can obtain a closed equation for the particle-velocity gradient $\sigma=V / R$,

$$
\dot{\sigma}=-\sigma^{2}-\tau^{-1}[\sigma-s(t)] .
$$

The Lyapunov exponent of particle trajectories can be obtained from Eq. (13) as an ensemble average $\langle\sigma\rangle$ over different realizations of the noise. Equations for the stationary distribution of $x=\sigma+1 /(2 \tau)$ are easily obtained with the same procedure adopted to derive Eqs. (9) and (10),

$$
\left[\left(\frac{1}{4 \tau^{2}}-x^{2}+\frac{s_{0}}{\tau}\right) p\right]_{x}+q_{x}=0,
$$




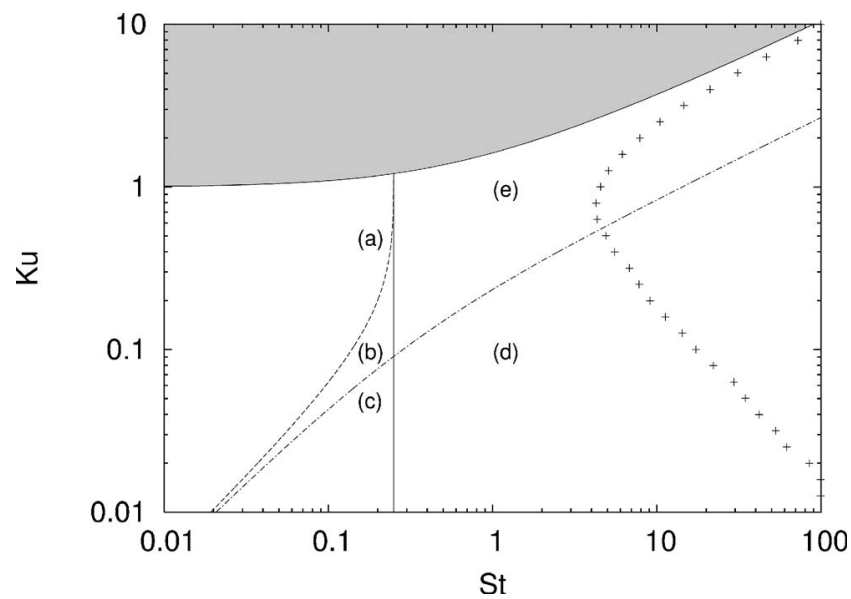

FIG. 1. Parameter space (St, Ku). Shape transitions in the probability density function (PDF) of $x$ occur for $\mathrm{St}=\mathrm{St}^{*}=1 / 4$ (vertical solid line), $\tilde{m}=1$ (dashed line), and $m=1$ (dash-dotted line). Labels refers to the PDFs shown in Figs. 2 and 3. The gray region is not accessible by the model because $\mathrm{Ku}$ does not fulfill condition (12). Crosses represent the boundary of the chaotic region.

$$
\left[\left(\frac{1}{4 \tau^{2}}-x^{2}-\frac{s_{0}}{\tau}\right) q\right]_{x}+\left(\frac{s^{2}-s_{0}^{2}}{\tau^{2}}\right) p_{x}+\nu q=0,
$$

where $q(x)=\int \tilde{s} p(x, \widetilde{s}) d \widetilde{s} / \tau$ and $p(x, \widetilde{s})$ is the joint stationary probability density function (PDF) of $x$ and $\tilde{s}$. From the first equation one obtains $q=-\left[C+\left(1 / 4 \tau^{2}-x^{2}+s_{0} / \tau\right) p\right]$, where $C$ is the mean flux of $x$, and finally

$$
\begin{aligned}
& {\left[\frac{s^{2}}{\tau^{2}}-\left(\frac{1}{4 \tau^{2}}-x^{2}\right)^{2}\right] p_{x}+\left[(4 x-\nu)\left(\frac{1}{4 \tau^{2}}-x^{2}\right)-\nu \frac{s_{0}}{\tau}\right] p} \\
& +C(2 x-\nu)=0 .
\end{aligned}
$$

The asymptotic behavior $p \sim C / x^{2}(C>0)$ for large $|x|$ gives the probability of strong particle velocity gradients, and is therefore related to the probability of shocks. Different solutions are found in the small-Stokes-number and largeStokes-number regime.

\section{A. Small Stokes number}

When $\mathrm{St}<\mathrm{St}^{*}$, the unique positive integrable solution of Eq. (16) is obtained under condition of zero flux $(C=0)$,

$$
p=C_{1} \frac{(w-x)^{m-1}(x-\widetilde{w})^{\tilde{m}-1}}{(w+x)^{m+1}(x+\widetilde{w})^{\tilde{m}+1}}, \quad x \in(\widetilde{w}, w),
$$

and zero otherwise, where

$$
\begin{array}{ll}
w=\sqrt{1+4 s \tau} / 2 \tau, & m=(\nu+\Delta \nu) / 4 w, \\
\widetilde{w}=\sqrt{1-4 s \tau} / 2 \tau, \quad \tilde{m}=(\nu-\Delta \nu) / 4 \widetilde{w} .
\end{array}
$$

The solution is localized in the compact interval $(\widetilde{w}, w)$. Its shape is determined by the values of $m$ and $\tilde{m}$. For $\tilde{m}$ $<1$ (low frequency) the PDF is peaked around the two border values. For $m<1<\tilde{m}$ (intermediate frequency) it vanishes at $\tilde{w}$, and finally when $m>1$ (high frequency) it van-

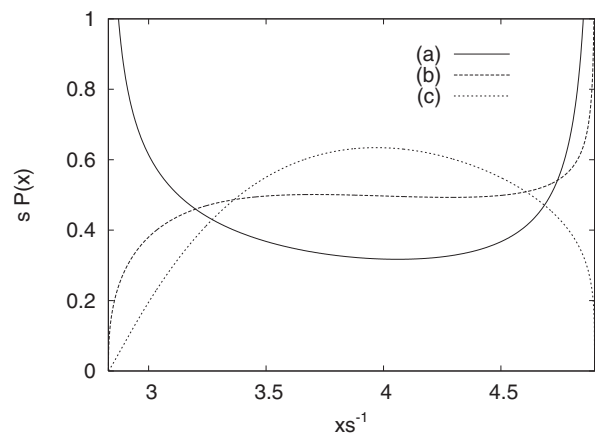

FIG. 2. PDF of $x$ in the small Stokes regime $(\mathrm{St}=1 / 8)$ for different value of the Kubo number: $\mathrm{Ku}=1 / 8(a), \mathrm{Ku}=1 / 16(b)$, and $\mathrm{Ku}=1 / 24(c)$.

ishes both at $\tilde{w}$ and $w$ (see Fig. 2). Indeed, when $\mathrm{St}<\mathrm{St}^{*}$, the solution of the linear system for $(R, V)$ oscillates between two asymptotes $V=(w-1 / 2 \tau) R$ and $V=(\widetilde{w}-1 / 2 \tau) R$ according to the sign of the noise. If the noise frequency is low the system has enough time to become close to the two asymptotes and the PDF is peaked around them. Conversely, when the sign of the noise switches frequently, the system does not have enough time to reach the asymptotes and oscillates rapidly around the mean value.

\section{B. Large Stokes number}

When $\mathrm{St}>\mathrm{St}^{*}$ the solution of Eq. (16) consists of two different parts,

$$
p(x)=C p_{1}(x)+C_{2} p_{2}(x) .
$$

The first one is the solution of Eq. (16) with $C=1$,

$$
\begin{aligned}
p_{1}= & \frac{|w-x|^{m-1}}{|w+x|^{m+1}\left(x^{2}+\widetilde{w}^{2}\right)} e^{n[\arctan (x / \widetilde{w})]} \\
& \times \int_{-w}^{x} d y \frac{|w+y|^{m+1}(\nu-2 y)}{|y-w|^{m-1}\left(w^{2}-y^{2}\right)} e^{-n[\arctan (y / \widetilde{w})]},
\end{aligned}
$$

while the second one is the right tail of the solution of the homogeneous (fluxless) equation

$$
p_{2}=\frac{|w-x|^{m-1}}{|w+x|^{m+1}\left(x^{2}+\tilde{w}^{2}\right)} e^{n[\arctan (x / \widetilde{w})]} I_{w, \infty}(x) .
$$

Here $I_{w, \infty}(x)$ is the characteristic function (indicator) of the interval $(w, \infty)$, and

$$
\begin{array}{ll}
w=\sqrt{4 s \tau+1} / 2 \tau, & m=(\nu+\Delta \nu) / 4 w, \\
\widetilde{w}=\sqrt{4 s \tau-1} / 2 \tau, \quad n=(\nu-\Delta \nu) / 2 \widetilde{w} .
\end{array}
$$

Conditions for determining $C$ and $C_{2}$ are

$$
\int p(x) d x=1, \quad \int q(x) d x=0 .
$$

These conditions guarantee the continuity of the PDF in the limit $\mathrm{St} \rightarrow \mathrm{St}^{*}$ (see the Appendix). The PDF obtained in the 


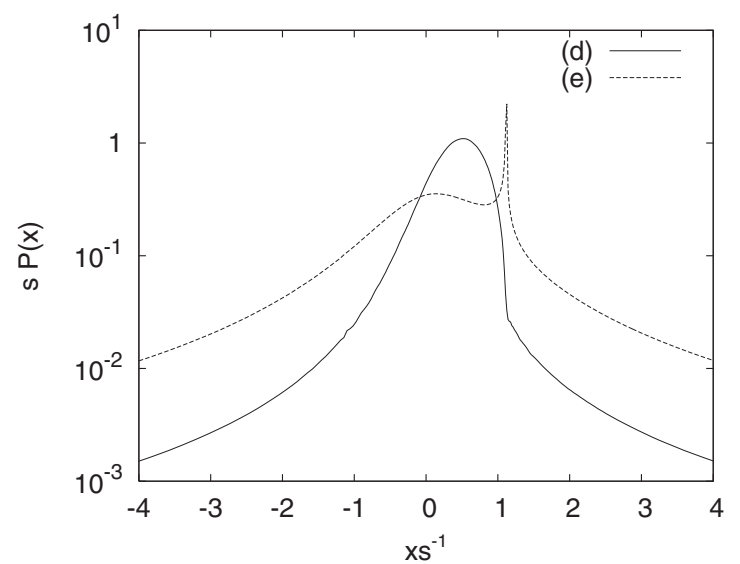

FIG. 3. PDF of $x$ in the large Stokes regime $(\mathrm{St}=1)$ for different values of Kubo number, $\mathrm{Ku}=0.1(d), \mathrm{Ku}=1(e)$.

regime $\mathrm{St}>\mathrm{St}^{*}$ is extended over all real $x$, with power-law tails $p \sim C / x^{2}$ for large $|x|$ that are due to shocks, which occur for large negative values of $s(t)$. For short-correlated noise $(m>1)$, the PDF is characterized by an asymmetric core localized between $-w$ and $w$. When $m<1$ a singular peak arises at $x=w$ (see Fig. 3). This behavior is easily understood in term of the solution of the linear system for $(R, V)$. When $s(t)=+s$ the solution converges toward the asymptote $V=\alpha R=(w-1 / 2 \tau) R$. This produces the peak at $x$ $=w$, provided that the correlation time of the noise is long enough $(m<1)$ to become close to the asymptote. The large Stokes number regime is hence characterized by an infinite series of shocks alternated to "quiet" phases in which the particle-velocity gradient relaxes toward $\alpha$.

The schematic in Fig. 1 summarizes the different regions in the parameter space $(\mathrm{St}, \mathrm{Ku})$ where shape transitions occurs in the PDF of $x$.

\section{LYAPUNOV EXPONENTS}

The Lyapunov exponent of inertial-particle trajectories can be written in terms of the mean value $\bar{x}$ as $\lambda=\bar{x}-1 / 2 \tau$. For $\mathrm{St}<\mathrm{St}^{*}$ the mean value $\bar{x}$ can be written as

$$
\bar{x}=\widetilde{w} \frac{(w-\widetilde{w}) \widetilde{m}}{(m+\widetilde{m})} \times \frac{F_{1}(\widetilde{m}+1, m+1, \widetilde{m}+1, \widetilde{m}+m+1, u, v)}{F_{1}(\widetilde{m}+, m+1, \widetilde{m}+1, \widetilde{m}+m, u, v)},
$$

where

$$
u=\frac{\widetilde{w}-w}{\widetilde{w}+w}, \quad v=\frac{\widetilde{w}-w}{2 \widetilde{w}},
$$

and $F_{1}$ is the hypergeometric function of two variables [25]. The behavior of the Lyapunov exponent as a function of the Stokes and Kubo numbers is shown in Fig. 4. The Lyapunov exponent is negative for small Stokes, and decreases approximatively as $\lambda s^{-1} \sim-\mathrm{Ku}$ at increasing $\mathrm{Ku}$ numbers. In the limit $\mathrm{St} \rightarrow 0$ it recovers the actual value for fluid tracers [20],

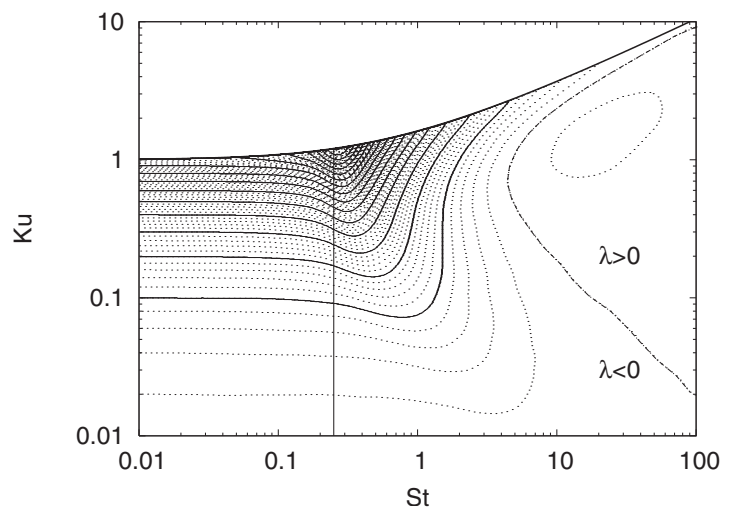

FIG. 4. The isolines of the Lyapunov exponent $\lambda$ on the plane of Stokes and Kubo numbers. The isolines are spaced every $0.02 \mathrm{~s}$ (dotted and solid lines). The boundary of the chaotic region $(\lambda$ $>0)$ is represented by the dot-dashed line.

$$
\lambda_{0} \equiv \lim _{\tau \rightarrow 0} \lambda=-s^{2} / \nu
$$

Notice that fluid tracers are always in the aggregation regime within this model, as signaled by the negative value of $\lambda_{0}$. A sharp negative minimum $\lambda=-2 s$ is found for $\mathrm{St}=1 / 4$ at $\mathrm{Ku}=(\sqrt{2}+1) / 2$. It corresponds to the maximum aggregation of the particle. As the correlation time of the flow decreases, the minimum becomes less pronounced, and it moves to larger Stokes numbers. A region of positive Lyapunov exponents is present in the parameter space for $\mathrm{St} \geq 4.3$. The isoline of vanishing Lyapunov exponent which border this region represent the transition from the strong clustering regime $(\lambda<0)$ to the chaotic regime $(\lambda>0)$. Indeed, Fig. 5 shows that, as Stokes number increases, the interval of Kubo numbers appears where the Lyapunov exponent grows, and eventually becomes positive. This can be understood as follows: to achieve an effective mixing the correlation time of the fluid gradients must be long enough to provide substantial stretching of particle trajectories, but not too long, to avoid particle segregation in compressing regions. Therefore the chaotic region is confined in a window of $\mathrm{Ku}$ numbers

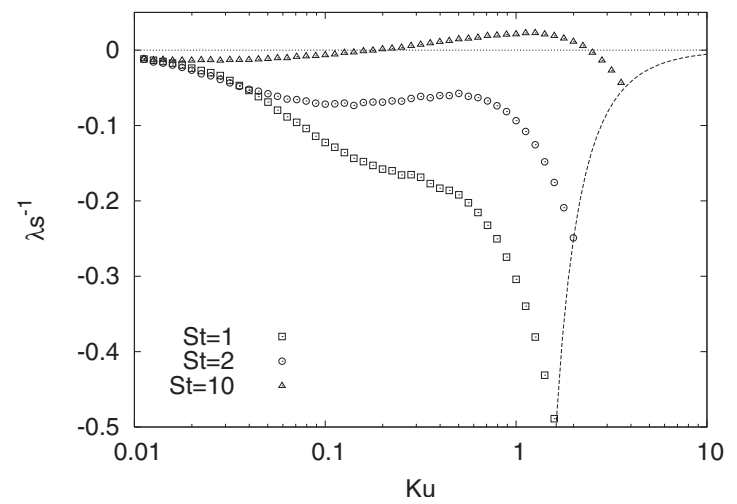

FIG. 5. The Lyapunov exponent $\lambda$ as a function of the Kubo number. An interval of positive Lyapunov exponents appears for $\mathrm{St} \gtrsim 4.3$. The dashed line represents the asymptotic behavior for $\mathrm{Ku}=\mathrm{Ku}^{*}$. 
between a lower and an upper bound determined by stretching efficiency and particle trapping respectively.

Let us now consider the behavior of the Lyapunov moments $\gamma_{n}$, defined as $\left\langle R^{n}\right\rangle \sim \exp \left(\gamma_{n} t\right)$. The evolution of $\left\langle R^{n}\right\rangle$, for $n$ positive integer, is determined by a closed system of the $2(n+1)$ equation,

$$
\begin{aligned}
\frac{d}{d t}\left\langle R^{n-i} V^{i}\right\rangle= & (n-i)\left\langle R^{n-i-1} V^{i+1}\right\rangle-\frac{i}{\tau}\left\langle R^{n-i} V^{i}\right\rangle \\
& +\frac{i}{\tau}\left(\widetilde{s}+s_{0}\right)\left\langle R^{n-i+1} V^{i-1}\right\rangle, \\
\frac{d}{d t}\left\langle\widetilde{s} R^{n-i} V^{i}\right\rangle= & (n-i)\left\langle\widetilde{s} R^{n-i-1} V^{i+1}\right\rangle-\left(\frac{i}{\tau}+\nu\right)\left\langle\widetilde{s} R^{n-i} V^{i}\right\rangle \\
& -\frac{i}{\tau} s_{0}\left\langle\widetilde{s} R^{n-i+1} V^{i-1}\right\rangle,
\end{aligned}
$$

where $i=0,1, \ldots, n$. The $n$th Lyapunov moment $\gamma_{n}$ is the largest solution of the $2(n+1)$ th equation

$$
\operatorname{det}\left[\begin{array}{ll}
\mathbf{A} & \mathbf{C} \\
\mathbf{D} & \mathbf{B}
\end{array}\right]=0
$$

$\mathbf{A}$ and $\mathbf{B}$ are tridiagonal matrices with the elements

$$
\begin{gathered}
A_{i, i}=\gamma_{n}+i / \tau, \quad i=1, n, \\
A_{i, i-1}=-i s_{0} / \tau, \quad i=2, n, \\
A_{i, i+1}=i-n, \quad i=1, n-1, \\
B_{i, i}=\gamma_{n}+\nu+i / \tau, \quad i=1, n, \\
B_{i, i-1}=i s_{0} / \tau, \quad i=2, n, \\
B_{i, i+1}=i-n, \quad i=1, n-1,
\end{gathered}
$$

and $\mathbf{C}$ and $\mathbf{D}$ are subdiagonal matrices with the elements $C_{i, i-1}=-i / \tau$ for $i=2, n$ and $D_{i, i-1}=i\left(s_{0}^{2}-s^{2}\right) / \tau$ for $i=2, n$. In the limit $\mathrm{St} \rightarrow 0$ one recovers the Lyapunov moments of fluid tracers [20],

$$
\gamma_{n}=\sqrt{\left(\frac{\nu}{2}\right)^{2}+s^{2}\left(n^{2}-n\right)}-\frac{\nu}{2} .
$$

Notice that for fluid tracers the sign of the separation $R$ is preserved, and hence the exponents $\gamma_{n}$ coincide with the exponents $\widetilde{\gamma}_{n}$ defined by $\left\langle|R|^{n}\right\rangle \sim \exp \left(\widetilde{\gamma}_{n} t\right)$. The asymptotic linear behavior of $\gamma_{n}$ for large $n$ is the hallmark of the presence of an upper bound for velocity gradients.

\section{A. Short-correlated flows}

Let us now examine the limit of short-correlated flow. The limit $\nu \rightarrow \infty$ must be taken keeping constant $s^{2} / \nu=D$, in order to recover $\delta$-correlated noise fluctuations $\left\langle s(t) s\left(t^{\prime}\right)\right\rangle$ $=2 D \delta\left(t-t^{\prime}\right)$. In other words, while Kubo number $\mathrm{Ku}=s / \nu$ tends to zero, Stokes number $\mathrm{St}=s \tau$ must grow, so that the product

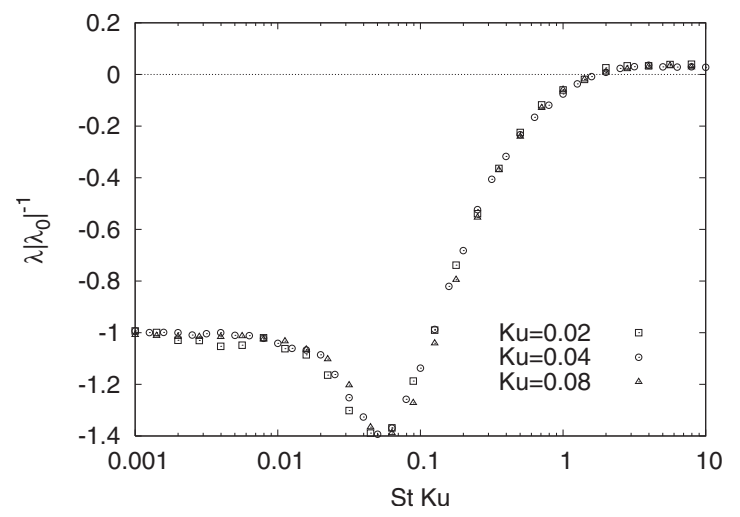

FIG. 6. Lyapunov exponent $\lambda$ in the short-correlated limit.

$$
\mathrm{Ku} \mathrm{St}=s^{2} / \nu \tau=D \tau,
$$

remains constant. In this sense the short-correlated limit for inertial particles correspond always to the large-inertia case. In the $\delta$-correlated limit the relevant time scale associated to fluid velocity gradients is given by the Lyapunov exponent of fluid tracers $\lambda_{0}=-s^{2} / \nu=-D$. This is confirmed by the collapse of particle Lyapunov exponents in the short-correlated limit once their intensity and Stokes times are rescaled with $\left|\lambda_{0}\right|$ (see Fig. 6). A noticeable minimum is observed for $\left|\lambda_{0}\right| \tau=\mathrm{Ku} \mathrm{St} \simeq 0.05$ and a transition to chaos, i.e., from negative to positive $\lambda$, occurs for $\mathrm{KuSt} \gtrsim 1.6$. These features are in qualitative agreement with previous analytic and theoretical results obtained in the framework of $\delta$-correlated flows $[14,18]$. Notice that in those studies Gaussian statistics is assumed for velocity gradients, at variance with our model in which only the two values $\pm s$ are allowed. Therefore quantitative details such as the exact position of the minimum can be different.

We remark that in the short-correlated asymptotics, the fluid-tracers limit becomes singular. Indeed one has

$$
\lim _{\nu \rightarrow \infty} s_{0}=\lim _{\nu \rightarrow \infty}-\frac{s^{2}}{\nu(1+\nu \tau)}= \begin{cases}-D & \tau=0, \\ 0 & \tau>0,\end{cases}
$$

which signals that fluid tracers are still preferentially attracted by regions of ongoing compression, while inertial particles with arbitrary finite $\tau$ are not. Notice that in the limit $\nu \rightarrow \infty$ fluid velocity gradients becomes unbound because $s \rightarrow \infty$, and for fluid tracers we recover the quadratic behavior of Lyapunov moments $\gamma_{n}=D\left(n^{2}-n\right)$.

\section{B. Long-correlated flows}

Time correlation of fluid gradient is bounded by the condition (12). When $\mathrm{Ku}=\mathrm{Ku}^{*}$ the transition rate $\nu_{1}$ from $-s$ to $s$ vanishes, while the transition rate $\nu_{2}$ from $s$ to $-s$ reaches its maximum $\nu^{*}=(\sqrt{1+4 s \tau}-1) /(2 \tau)$. Particles initially seeded in expanding regions are gradually captured by contracting ones, where they remain trapped forever. Therefore the population of expanding regions decreases exponentially as 


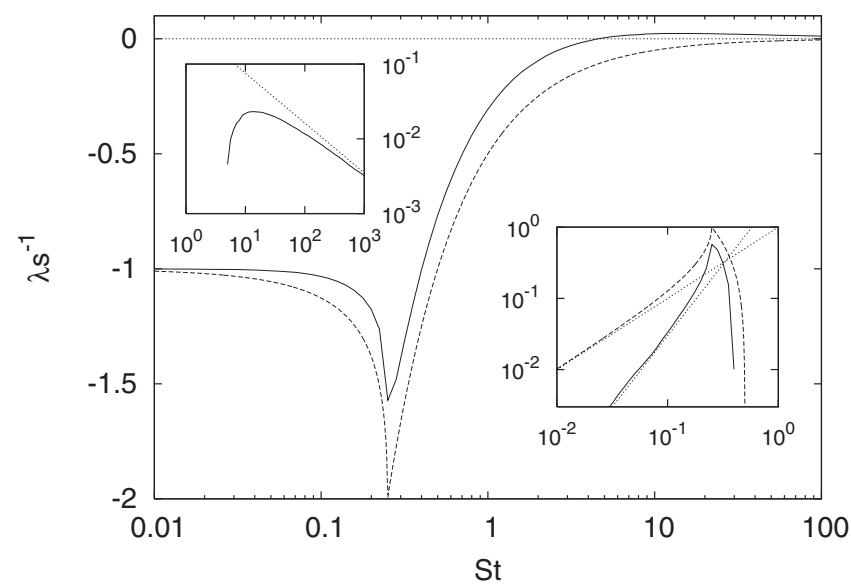

FIG. 7. Lyapunov exponent $\lambda$ for $\mathrm{Ku}=1$ (solid line) and on the line $\mathrm{Ku}=\mathrm{Ku}^{*}$ (dashed line), corresponding to the longest correlation of velocity gradients achievable within the model. Upper inset: asymptotic behavior for large Stokes number $\lambda s^{-1} \sim \mathrm{St}^{-2 / 3}$ (dotted line), $(\mathrm{Ku}=1)$. Lower inset: asymptotic behavior for small Stokes number $\left|\lambda-\lambda_{0}\right| s^{-1} \sim \mathrm{St}^{\zeta}$ (dotted line).

$$
P(t) \sim \exp \left(-\nu^{*} t\right)
$$

Moments of particle separations will hence evolve asymptotically according to

$$
\left\langle|R|^{n}\right\rangle \sim e^{n \alpha t} P(t)+(1-P(t)) e^{n \operatorname{Re}(\beta) t} f(\operatorname{Im}(\beta) t),
$$

where $\beta$ is given by Eq. (5) and $f(t)$ is $2 \pi$ periodic function. Notice that $\nu^{*}=\alpha$ and therefore for $n=1$ the decreasing fraction of particle in expanding regions is exactly balanced by the exponential growth of their separation. From Eqs. (34) and (35) one obtains the Lyapunov moments

$$
\begin{gathered}
\tilde{\gamma}_{n}=\alpha(n-1) \text { for } n \geqslant(\alpha-\operatorname{Re}(\beta))^{-1}, \\
\tilde{\gamma}_{n}=\operatorname{Re}(\beta) \text { for } n \leqslant(\alpha-\operatorname{Re}(\beta))^{-1} .
\end{gathered}
$$

In the limit of fluid tracers one obtains

$$
\begin{gathered}
\tilde{\gamma}_{n}=s(n-1) \quad \text { for } n \geqslant 1 / 2, \\
\tilde{\gamma}_{n}=-s n \quad \text { for } n \leqslant 1 / 2,
\end{gathered}
$$

in agreement with Eq. (31).

Finally, the Lyapunov exponent along the critical line $\mathrm{Ku}=\mathrm{Ku}^{*}$ is $\lambda=\left.\left(\partial \widetilde{\gamma}_{n} / \partial\right)\right|_{n=0}=\operatorname{Re}(\beta)$. In Fig. 7 we compare its behavior with that of the Lyapunov exponent along the line $\mathrm{Ku}=1$. For small Stokes number both of them recover the fluid-tracers limit $\lambda \rightarrow \lambda_{0}=-s$, but with different power-law behavior. On the line $\mathrm{Ku}=\mathrm{Ku}^{*}$ we have $\left|\lambda-\lambda_{0}\right| s^{-1} \sim \mathrm{St}$, while for $\mathrm{Ku}=1$ we have $\left|\lambda-\lambda_{0}\right| s^{-1} \sim \mathrm{St}^{2}$ (see lower inset of Fig. 7). For large Stokes number Lyapunov vanishes as $\lambda s^{-1} \sim-\mathrm{St}^{-1}$ on the line $\mathrm{Ku}=\mathrm{Ku}^{*}$ and as $\lambda s^{-1} \sim \mathrm{St}^{-2 / 3}$ for $\mathrm{Ku}=1$ (see upper inset of Fig. 7). In between these two asymptotics a sharp minimum appears for $\mathrm{St}=\mathrm{St}^{*}$.

Notice that the asymptotic decay $\mathrm{St}^{-2 / 3}$, here shown for long-correlated flows, have been already predicted and observed also for $\delta$-correlated flows $[17,19]$. The agreement between these results confirms that in the large Stokes number asymptotics the role of time correlation becomes negligible and particles behave as if suspended in $\delta$-correlated-in-time flows.

\section{CONCLUSIONS}

We discussed the Lagrangian dynamics of inertial particles in a simple time-correlated compressible flow, in which fluid velocity gradients in the reference frame of the particle are modeled by a one-dimensional telegraph noise. In spite of its simplicity, the model allows one to take into account consistently the different Lagrangian weights of regions of ongoing compression and expansion, and it reproduces the phenomenon of trapping of particles in compressing regions for long-correlated flows.

The peculiar nature of the telegraph noise allows one to investigate analytically the effects of time correlation of velocity gradients on the chaoticity of particle trajectories, and to study the dependence on Stokes and Kubo numbers of Lyapunov exponents. We discussed both the asymptotics of long- and short-correlated flows, as well as the fluid-tracers limit.

For large Stokes number, a regime characterized by the formation of shocks, we found a chaotic region in parameter space $(\mathrm{St}, \mathrm{Ku})$, where the leading Lyapunov exponent becomes positive. Inertia is therefore responsible for a transition from a strong clustering regime, originated by the compressible nature of the flow, to a chaotic regime. The latter is observed in a range of Kubo numbers such that the time correlation of fluid gradients is long enough to provide substantial stretching, but not too long to cause particles trapped in compressing regions.

This simple model clearly does not allow one to reproduce all the complex phenomena which occur in a realistic flow. In particular it does not include the effects of preferential concentration in hyperbolic regions, which are responsible for the increase of chaoticity of inertial particle trajectories in turbulent flows [10]. A two-dimensional extension of the model would be required for investigating these effects. Further, we remind the reader that the Lyapunov statistics is able to describe the clustering of inertial particles at the dissipative scales of turbulent flows, but does not allow one to characterize the structures which are observed in particle distribution at scales within the turbulent inertial range [26].

Note that the substitution $R=\Psi \exp (-t / 2 \tau)$ turns Eq. (2) into the Schrödinger equation, $\ddot{\Psi}-s \Psi / \tau=\Psi / 4 \tau^{2}$, with space replacing time. The telegraph noise model for the Schrödinger equation was used by Bendesrkii and Pastur [27], who applied the methods of [28] to evaluate the density of states.

\section{ACKNOWLEDGMENTS}

We are grateful to K. Gawędzki who called our attention to [27]. This work has been supported by the ONR Grant No. N00014-99-0042 and by the grant of the Israeli Science Foundation. 


\section{APPENDIX}

To prove the continuity of the $\mathrm{PDF}$ for $\mathrm{St}=\mathrm{St}^{*}$ we first notice that the limit $\mathrm{St} \rightarrow \mathrm{St}^{*}$ is equivalent to $\widetilde{w} \rightarrow 0$.

The limit $\widetilde{w} \rightarrow 0$ of the solution (17) in the small-Stokes number regime is $p(x)=C_{1} f(x) I_{0, w}(x)$, where

$$
f(x)=\frac{(w-x)^{m-1}}{(w+x)^{m+1} x^{2}} e^{-(\nu-\Delta \nu) / 2 x},
$$

and $I_{0, w}(x)$ is the characteristic function of the interval $(0, w)$.

To study the limit of the solution (19), let us consider the limits of $p_{1}(x)$ and $p_{2}(x)$ when $\widetilde{w} \rightarrow 0$. The latter is easily obtained as

$$
p_{2}(x) \sim e^{(\nu-\Delta \nu) \pi / 4 \tilde{w}} f(x) .
$$

Now let us rewrite $p_{1}(x)$ as

$$
\begin{aligned}
p_{1}= & \frac{|w-x|^{m-1}}{|w+x|^{m+1}\left(x^{2}+\tilde{w}^{2}\right)} e^{-n[\arctan (\widetilde{w} / x)]} \\
& \times \int_{-w}^{x} \frac{|w+y|^{m+1}(\nu-2 y) d y}{|y-w|^{m-1}\left(w^{2}-y^{2}\right)} e^{n[\arctan (\widetilde{w} / y)]} .
\end{aligned}
$$

As $\tilde{w} \rightarrow 0$, then $p_{1}(x)=O(1)$ if $x<0$ and

$$
p_{1}(x) \sim 2 e^{(\nu-\Delta \nu) \pi / 4 \widetilde{w}} \widetilde{w}^{2} f(x),
$$

for $x>0$. To obtain the last asymptotic one takes into account that the main contribution in the integral in Eq. (A3) is brought by the right small vicinity of $y=0$, say $(0, s)$, and apply

$$
\int_{0}^{s} \exp [-\arctan (y / \delta) / \delta] d y \sim \int_{0}^{s} \exp \left(-y / \delta^{2}\right) d y \sim \delta^{2}
$$

for all $s>0$ and small $\delta$.

The continuity of the solution of Eq. (16) in the limit $\widetilde{w}$ $\rightarrow 0$ is therefore guaranteed by the conditions

$$
C=\frac{C_{1}}{2 \widetilde{w}^{2} e^{(\nu-\Delta \nu) \pi / 4 \tilde{w}}}, \quad C_{2}=2 C \widetilde{w}^{2} .
$$

This conditions are indeed equivalent to the normalizations conditions

$$
\int p(x) d x=1, \quad \int q(x) d x=0,
$$

where $q=-\left[C+\left(1 / 4 \tau^{2}+s_{0} / \tau-x^{2}\right) p\right]$.

To show this we notice that Eq. (16) can be written as

$$
\left\{\left[\frac{s^{2}}{\tau^{2}}-\left(\frac{1}{4 \tau^{2}}-x^{2}\right)^{2}\right] p+C x^{2}\right\}_{x}+\nu q=0 .
$$

It follows that

$$
\int_{-x}^{x} q(y) d y=-\frac{1}{\nu}\left[\frac{s^{2}}{\tau^{2}}-\left(\frac{1}{4 \tau^{2}}-x^{2}\right)^{2}\right][p(x)-p(-x)] .
$$

Thus, the second condition in Eq. (A7) is equivalent to

$$
\lim _{x \rightarrow \infty} x^{4}[p(x)-p(-x)]=0 .
$$

According to Eq. (19) $p(x)$ is written as the sum of $p_{1}$ and $p_{2}$, whose asymptotic behavior is

$$
p_{1}(x) \sim \frac{1}{x^{2}}+\frac{P_{1}^{+}}{x^{4}}, \quad x \rightarrow \infty, \quad p_{1}(x) \sim \frac{1}{x^{2}}+\frac{P_{1}^{-}}{x^{4}}, \quad x \rightarrow-\infty,
$$

and

$$
p_{2}(x) \sim \frac{P_{2}^{+}}{x^{4}}, \quad x \rightarrow \infty, \quad p_{2}(x) \sim \frac{P_{2}^{-}}{x^{4}}, \quad x \rightarrow-\infty .
$$

Notice that $P_{2}^{-}=0, P_{2}^{+}=e^{(\nu-\Delta \nu) \pi / 4 \widetilde{w}}$. Thus Eq. (A10) becomes

$$
C_{2}=\frac{C\left(P_{1}^{+}-P_{1}^{-}\right)}{P_{2}^{+}} \text {. }
$$

In the limit $\tilde{w} \rightarrow 0$,

$$
P_{1}^{-}=o\left(P_{1}^{+}\right), \quad P_{1}^{+} \sim 2 \widetilde{w}^{2} P_{2}^{+},
$$

and we obtain

$$
C_{2} \sim 2 \widetilde{w}^{2} C,
$$

which implies the continuity of the PDF. Normalization of $p(x)$ in the limit $\widetilde{w} \rightarrow 0$ is equivalent to the first condition in Eq. (A6).
[1] M. Maxey, J. Fluid Mech. 174, 441 (1987).

[2] E. Balkovsky, G Falkovich, and A. Fouxon, Phys. Rev. Lett. 86, 2790 (2001).

[3] J. C. Sommerer and E. Ott, Science 259, 335 (1993).

[4] J. Schumacher and B. Eckhardt, Phys. Rev. E 66, 017303 (2002).

[5] J. Bec, K. Gawedzki, and P. Horvai, Phys. Rev. Lett. 92, 224501 (2004).

[6] G. Boffetta, J. Davoudi, B. Eckhardt, and J. Schumacher, Phys. Rev. Lett. 93, 134501 (2004).
[7] J. Bec, Phys. Fluids 15, L81 (2003).

[8] G. Falkovich and A. Pumir, Phys. Fluids 16, L47 (2004).

[9] K. Duncan, B. Mehlig, S. Östlund, and M. Wilkinson, Phys. Rev. Lett. 95, 240602 (2005).

[10] J. Bec, L. Biferale, G. Boffetta, M. Cencini, S. Musacchio, and F. Toschi, Phys. Fluids 18, 091702 (2006).

[11] M. Chertkov, I. Kolokolov, and M. Vergassola, Phys. Rev. Lett. 80, 512 (1998).

[12] G. Falkovich, K. Gawȩdzki, and M. Vergassola, Rev. Mod. Phys. 73, 913 (2001). 
[13] J. M. Deutsh, J. Phys. A 18, 1449 (1985).

[14] M. Wilkinson and B. Mehlig, Phys. Rev. E 68, 040101(R) (2003).

[15] B. Mehlig and M. Wilkinson, Phys. Rev. Lett. 92, 250602 (2004).

[16] B. Mehlig, M. Wilkinson, K. Duncan, T. Weber, and M. Ljunggren, Phys. Rev. E 72, 051104 (2005).

[17] J. Bec, M. Cencini, and R. Hillerbrand, Physica D 222, 11 (2007).

[18] S. A. Derevyanko, G. Falkovich, K. Turitsyn, and S. Turitsyn, J. Turbul. 8, 1 (2007).

[19] A. Fouxon and P. Horvai, e-print arXiv:nlin.CD/0704.3893v1.

[20] G. Falkovich and M. Martins-Afonso, Phys. Rev. E 76, 026312 (2007).
[21] M. R. Maxey and J. Riley, Phys. Fluids 26, 883 (1983).

[22] G. Falkovich, A. Fouxon, and M. Stepanov, Nature (London) 419, 151 (2002).

[23] M. Wilkinson, B. Mehlig, and V. Bezuglyy, Phys. Rev. Lett. 97, 048501 (2006).

[24] V. E. Shapiro and V. M. Loginov, Physica A 91, 563 (1978).

[25] I. S. Gradshteyn and I. M. Ryzhik, Table of Integrals, Series, and Products, 5th ed. (Academic, New York, 1994), pp. 1204.

[26] J. Bec, L. Biferale, M. Cencini, A. Lanotte, S. Musacchio, and F. Toschi, Phys. Rev. Lett. 98, 084502 (2007).

[27] M. M. Benderskii and L. A. Pastur, Sov. Phys. JETP 30, 158 (1970).

[28] H. L. Frisch and S. P. Lloyd, Phys. Rev. 120, 1175 (1960). 